\title{
Comparing Differential Gene Expression in Chronic Traumatic Encephalopathy, Parkinson's Disease, and Bipolar Disorder
}

Francia Victoria De Los Reyes and Carina Villamayor

University of the East Ramon Magsaysay Memorial Medical Center College of Medicine, Quezon City, Philippines

\section{ABSTRACT}

Introduction. Chronic traumatic encephalopathy (CTE) is a progressive neurodegenerative disorder that is defined, neuropathologically, by the presence of aggregated hyperphosphorylated tau in the neurons and astrocytes of the perivascular area that is located deep in the cerebral sulci. The lesion is associated with repetitive brain trauma, from the spectrum of asymptomatic subconcussive head injury to grossly identifiable features of concussion. Although the diagnostic neuropathology of CTE is well-characterized, the precise mechanism that causes this to occur in CTE is not yet clearly elucidated. The features of hyperphosphorylated tau in CTE is quite similar with Alzheimer's Disease (AD), as is the reduced expression of certain genes that are required to dephosphorylate tau, which is the putative culprit in the generation of amyloid aggregates and hyperphosphorylated tau.' In comparison, Parkinson's Disease (PD) is a neurodegenerative disease that is caused by accumulation of misfolded alpha-synuclein ( $\alpha$-syn) that causes the formation of intraneuronal Lewy Body aggregates. The pattern of accumulation for a-syn involves the olfactory bulb and the gut with progressive involvement of the posterior part of the brain. ${ }^{2}$ Despite establishing the presence of two different intraneuronal inclusions for CTE and PD, contact sports associated with the clinical spectrum of CTE has been shown to present with Parkinsonian features along with dementia. Mood disorders has been reported to occur in patients with these neurologic conditions. Several studies have documented that patients had a previous experience of traumatic brain injury prior to the diagnosis of Bipolar Disorder (BD). A review of electronic literature suggested that having an earlier diagnosis of BD increased the likelihood of having a diagnosis of PD in the future..$^{3,4}$

Objectives. This research aimed to compare the over- and underexpressed genes in cases with Parkinson's Disease (PD), cases with Bipolar Disorder (BD), and cases with Chronic Traumatic Encephalopathy (CTE) versus normal controls. This was done to determine if parallel overexpression in certain genes may indicate the possible association at the level of gene expression. Identifying similar RNA sequence establishing gene expression may provide an insight to the relationship of the diseases in terms of pathobiological behavior. Determining the similar over- or underexpression pattern may provide an insight on the common pathobiologic mechanisms that may be the reason for the three disorders being associated by way of pre-morbid or co-morbid condition.

Methodology. Transcripts from the public domain archive of the NCBI SRA were identified for the RNA sequence (RNAseq) of interest using the search string "Chronic Traumatic Encephalopathy", "Bipolar Disorder", and "Parkinson". Only public domain transcriptome files of post-mortem brain samples labeled as RNAseq data extracted thru the Illumina platform that have a paired normal control were selected. A total of ten (10) cases for each disorder and thirty (30) normal subjects for control in the NCBI SRA RNAseq database with a whole exome sequence file that was available for public domain use was utilized for differential gene expression analysis. $6.7,8$

Results and Discussion. Among 21,122 identified genes from the RNAseq, the analysis was able to identify 26 genes exhibiting increased expression of up to >15 log2 fold change among cases with CTE, PD, and BD compared with normal controls. In contradistinction, only 6 well-described genes exhibited a decreased

ISSN 2507-8364 (Online)

Printed in the Philippines.

Copyright $@ 2020$ by the PJP.

Received: 27 March 2020.

Accepted: 19 June 2020.

Published online first: 22 June 2020.

https://doi.org/10.21141/PJP.2020.04

Corresponding author: Francia Victoria A. De Los Reyes, MD E-mail: kaidelosreyes@gmail.com

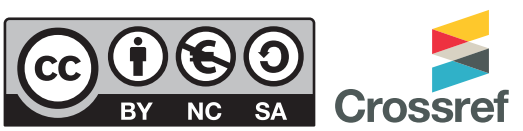
expression among cases with CTE and BD compared to normal controls. However, there were no identified genes that exhibited underexpression in cases with PD compared with normal controls.

The identification of parallel gene overexpression among the CTE, BD, and PD groups with respect to structural integrity, cellular metabolism, homeostasis, and apoptosis may indicate a common pathway that have been initiated as part of the response to maintain tissue function or as a consequence of the underlying pathobiologic mechanism that caused the primary lesion. 
In comparison, the underexpressed genes detected in the CTE and BD cases compared to the normal controls and the PD cases may indicate the lack of genes that have a role in repressing the mRNA for protein coding.

Conclusion. The overexpression of genes responsible for homeostasis, regulation of inflammation, balance of apoptosis and anti-apoptosis, and maintenance of structural integrity among the CTE, BD, and PD groups indicate that there is an interrelated mechanism that serves converging pathways as part of the response to lesional- forming structures in the brain. The goal of studies such as this is to have a better understanding on the common pathways that explain the interrelatedness of brain disorders and the putative mechanism for being co-morbid and pre-morbid conditions of each other.

Key words: differential gene expression, RNAseq, Chronic Traumatic Encephalopathy, Bipolar Disorder, Parkinson's Disease

\section{INTRODUCTION}

Chronic traumatic encephalopathy (CTE) is a progressive neurodegenerative disorder that is defined, neuropathologically, by the presence of aggregated hyperphosphorylated tau in the neurons and astrocytes of the perivascular area that is located deep in the cerebral sulci. The lesion is associated with repetitive brain trauma, from the spectrum of asymptomatic subconcussive head injury to grossly identifiable features of concussion. Although the diagnostic neuropathology of CTE is wellcharacterized, the precise mechanism that causes this to occur in CTE is not yet clearly elucidated. The features of hyperphosphorylated tau in CTE is quite similar with Alzheimer's Disease (AD), as is the reduced expression of certain genes that are required to dephosphorylate tau, which is the putative culprit in the generation of amyloid aggregates and hyperphosphorylated tau. ${ }^{1}$

In comparison, Parkinson's Disease (PD) is a neurodegenerative disease that is caused by accumulation of misfolded alpha-synuclein ( $\alpha$-syn) that causes the formation of intraneuronal Lewy Body aggregates. The pattern of accumulation for $\alpha$-syn involves the olfactory bulb and the gut with progressive involvement of the posterior part of the brain. ${ }^{2}$

Despite establishing the presence of two different intraneuronal inclusions for CTE and PD, contact sports associated with the clinical spectrum of CTE has been shown to present with Parkinsonian features along with dementia. Mood disorders has been reported to occur in patients with these neurologic conditions. Several studies have documented that patients had a previous experience of traumatic brain injury prior to the diagnosis of Bipolar Disorder (BD). A review of electronic literature suggested that having an earlier diagnosis of $\mathrm{BD}$ increased the likelihood of having a diagnosis of PD in the future. ${ }^{3,4}$ Newer studies in chronic mental illness have shown that patients diagnosed with $\mathrm{BD}$ exhibited aberrant aggregation of Disrupted-In-Schizophrenia 1 (DISC1) in post-mortem brain tissue, particularly in the cingulate cortex. ${ }^{5}$

The association of intraneuronal aggregates with the clinical presentation of the lesions, the presence of $\mathrm{BD}$ as a co-morbid or pre-morbid condition for both CTE and PD patients, and the relationship of CTE and PD, may lead to a possible association between these three lesions. The association may be in terms of increased or decreased gene expression of the patients compare to that of the normal population that provides an intra- and extracellular milieu to develop protein aggregates and manifest with the clinical symptoms.

\section{OBJECTIVES}

This research aimed to compare the over- and underexpressed genes in cases with Parkinson's Disease (PD), cases with Bipolar Disorder (BD), and cases with Chronic Traumatic Encephalopathy (CTE) versus normal controls. This was done to determine if parallel overexpression in certain genes may indicate the possible association at the level of gene expression. Identifying similar RNA sequence establishing gene expression may provide an insight to the relationship of the diseases in terms of pathobiological behavior. Determining the similar over- or underexpression pattern may provide an insight on the common pathobiologic mechanisms that may be the reason for the three disorders being associated by way of pre-morbid or co-morbid condition.

\section{METHODOLOGY}

Transcripts from the public domain archive of the NCBI SRA were identified for the RNA sequence (RNAseq) of interest using the search string "Chronic Traumatic Encephalopathy", "Bipolar Disorder", and "Parkinson's". Only public domain transcriptome files of post-mortem brain samples labeled as RNAseq data extracted thru the Illumina platform that have a paired normal control were selected. A total of ten (10) cases for each disorder and thirty (30) normal subjects for control in the NCBI SRA RNAseq database with a whole exome sequence file that was available for public domain use was utilized for differential gene expression analysis. ${ }^{6-8}$ Available transcriptome of post-mortem brain tissue from cases with CTE, cases with PD, cases with $\mathrm{BD}$, and normal controls were analyzed separately. Analysis was done using the Galaxy platform ${ }^{9}$ to extract the RNAseq of the identified cases from the European Nucleotide Archive (ENA). ${ }^{10}$ The RNAseq were compared to the reference Homo sapiens (human) genome Genome Reference Consortium Human Build 38 (hg38). ${ }^{11}$ Alignment of the RNAseq to the reference genome was done using HISAT2. ${ }^{12}$

The RNAseq was categorized per disease type with 10 PD, $10 \mathrm{CTE}, 10 \mathrm{BD}$, and 30 normal subjects that were compiled separately to generate a table to compare the counted gene 
expression per identified gene. ${ }^{13,14}$ Calculation of the $\log 2$ fold change of all the identified genes for normalization were done to determine which genes were overexpressed or underexpressed with doubling of the original value equal to a log2 fold change of 1 was done using $\mathrm{R}$ under RStudio with the assigned FDR adjusted p-value $<0.05 .{ }^{15,16}$

Comparison between the genes of patient groups with CTE, $\mathrm{PD}$, and $\mathrm{BD}$, with the cut off of overexpression assigned as $\geq 15 \log 2$ fold change across all patient categories, versus $\leq 0 \log 2$ fold change expression at FDR adjusted p-value $<0.05$ in normal controls identified the presence of overexpressed genes that significantly differed from normal controls. Comparison for gene underexpression in CTE, PD, and BD with normal controls with a difference of $1 \log 2$ fold change at FDR adjusted p-value $<0.05$ was also done. Calculation for statistically significant difference between the transcriptome of normal subjects versus CT, $\mathrm{PD}$, and $\mathrm{BD}$ were done using Mann-Whitney $\mathrm{U}$ with a p-value of $<0.01$.

\section{RESULTS AND DISCUSSION}

Among 21,122 identified genes from the RNAseq, the analysis was able to identify 26 genes exhibiting increased expression of up to $>15 \log 2$ fold change among cases with CTE, PD, and BD compared with normal controls. The genes have been observed to have an observable functional association based on worldwide gene co-expression database (Figure 1. Observed Co-expression of the Genes in Homo sapiens). It must be noted that, although not all genes have an outright direct connection in terms of their cellular processes, the network of overexpressed genes have a significantly greater interaction than expected (Figure 2. Network Interaction of the Genes in Homo sapiens). In contradistinction, only 6 well-described genes exhibited a decreased expression among cases with CTE and $\mathrm{BD}$ compared to normal controls. However, there were no identified genes that exhibited underexpression in cases with PD compared with normal controls. ${ }^{17}$
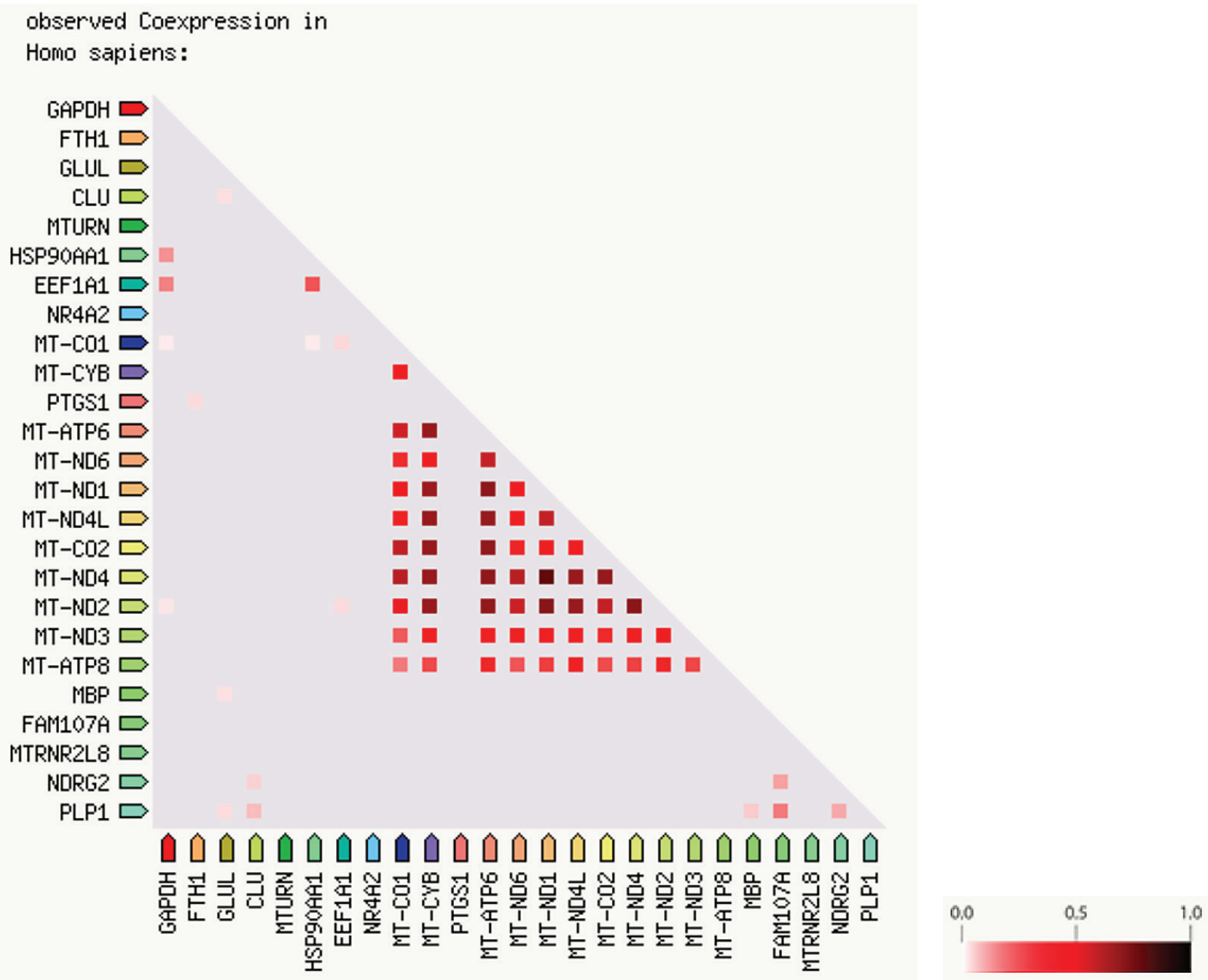

Figure 1. Co-expression predicts functional association: In the triangle matrix above, the intensity of color indicates the level of confidence that two proteins are functionally associated, given the overall expression data in the organism. Generated using STRING v11. https:/string-db.org/ 


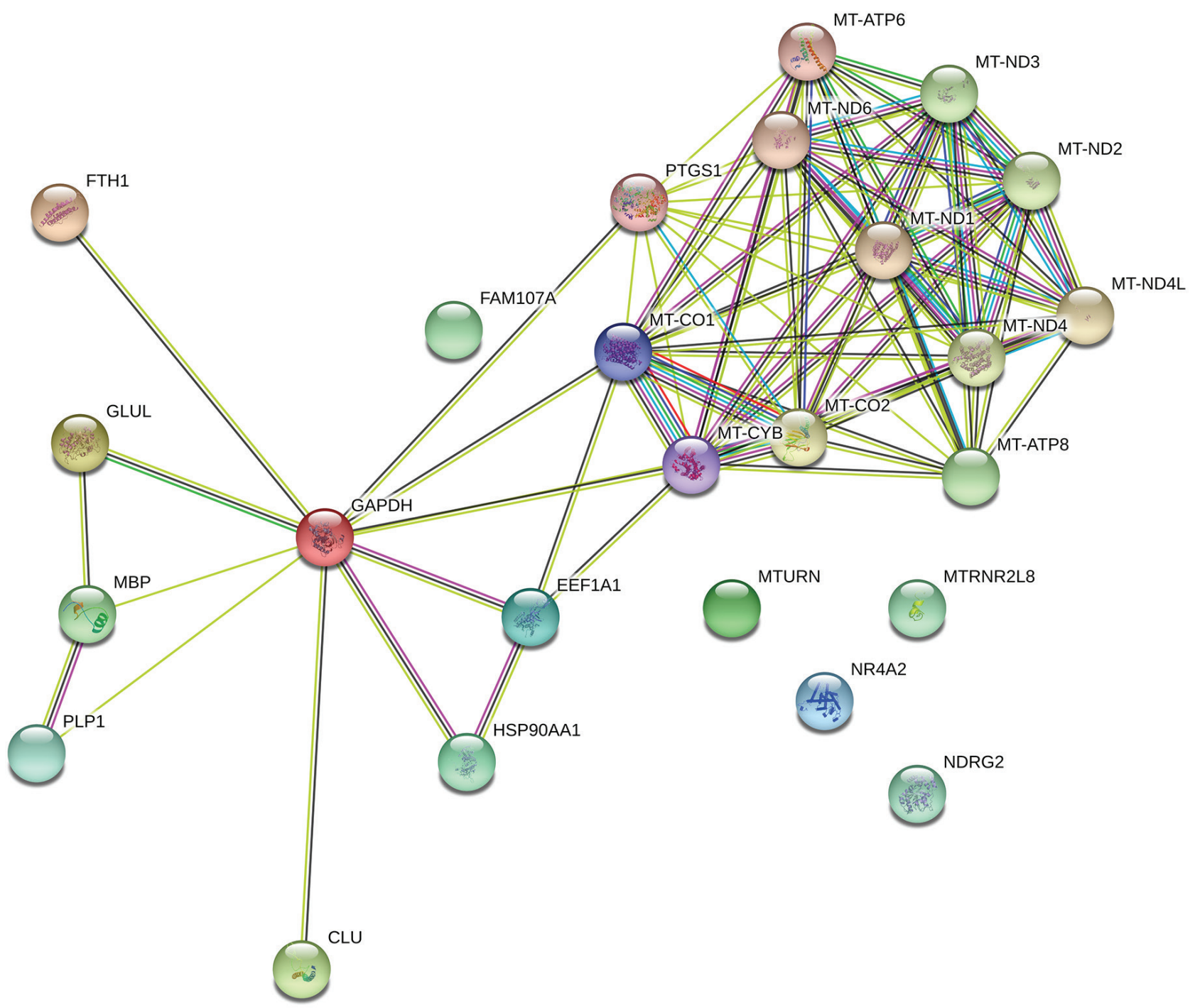

Figure 2. Each node (three-dimensional circle) represents the protein produced by a single, protein-coding gene locus identified in the study. The colored nodes indicate the query proteins which are products of the overexpressed genes. The filled nodes indicate that some of the protein structures are known or predicted. The connecting bars connote interactions that may be from predicted gene neighborhood, fusion, co-occurence, co-expression, protein homology or textmining. Generated using STRING v11. https:/string-db.org/

Among the genes with increased expression, FAM107A, or family with sequence similarity 107 member A, showed the most documented association with $\mathrm{BD}$ since high expression has been found in the dorsolateral prefrontal cortex of patients with $\mathrm{BD}$ and schizophrenia. ${ }^{18}$ The protein encoded by this gene has been responsible or the modulation of actin filamentous (F-actin) dynamics that contributes to synaptic and cognitive function at the level of the hippocampus. ${ }^{19}$

FTH1, or ferritin heavy chain 1, also has an increased expression, and this gene codes for the component of Ferritin protein. Ferritin functions as storage of iron in a soluble and nontoxic state. Previous studies have described an increase in Ferritin reactivity in the brains of PD patients. ${ }^{20}$

As for the regulation of apoptosis and structural integrity, RNR2 has been noted to have an anti-apoptotic function, ${ }^{21}$ while GAPDH has been reported in previous studies as an apoptosis initiator. ${ }^{22}$
CLU, or clusterin, has been described to encode for a protein that functions as a chaperone which is secreted in the cytosol under some stress conditions. Involvement has been described in processes such as cell death, tumor progression, and neurodegenerative disorders. ${ }^{23}$

HSP90AA1, or heat shock protein 90 alpha family class A member 1, has a product that consists of an inducible molecular chaperone that aids in proper protein-folding by use of an ATPase activity. ${ }^{24}$

Genes involved in neuronal development and growth regulation have also been identified in the overexpressed category, such as MTURN, NDRG2, GLUL, PLP1, RNR1/ NR4A2, MBP, and EEF1A1.

MTURN, which stands for maturin, and known as neural progenitor differentiation regulator homolog, has been known to represses NF-kappa-B transcriptional activity and has been documented in early neuronal development. ${ }^{25}$ 
NDRG2, from the NDRG family, was another overexpressed protein that was identified with neuronal growth involvement since the protein encoded by this gene is a cytoplasmic protein that may play a role in neurite outgrowth. ${ }^{26}$

GLUL, or glutamate-ammonia ligase, codes for a protein that has a role in ammonia and glutamate detoxification, acid-base homeostasis, cell signaling, and cell proliferation. ${ }^{27}$

PLP1, or proteolipid protein 1, encodes for a protein that has a function in ammonia and glutamate detoxification, acid-base homeostasis, cell signaling, and cell proliferation. ${ }^{28}$

RNR1/ NR4A2, or ribosomal 1/ nuclear receptor subfamily 4 , group A, member 2 , has been described as a regulator of insulin sensitivity and metabolic homeostasis. ${ }^{29}$

MBP, or myelin basic protein has been documented to play a role in regulating blood-brain barrier function since antibodies specific to MBP have been shown to increase blood-brain barrier permeability. ${ }^{30}$

EEF1A1, or eukaryotic translation elongation factor 1 alpha 1 , is a subunit of a protein that has been described to enzymatically deliver aminoacyl tRNAs to the ribosome. ${ }^{31}$

In terms of cellular metabolism, three functioned as part of cytochrome c oxidase components (COX1/ PTGS1, COX2, COX3). However, it has been shown that the key enzyme produced by COX1/ PTGS1 play a role in neuroinflammatory processes and COX2 has been responsible for mediating neuroprotection; ${ }^{32}$ while an increase in COX3 has been shown to increase astrocytic aerobic metabolism. ${ }^{33}$ Several other genes involved in oxidative phosphorylation and carbon handling have also been observed such as cytochrome c reductase complex (CYTB), ${ }^{34}$ part of the mitochondrial membrane ATP synthase (ATP6, ATP8), ${ }^{35}$ part of the NADH dehydrogenase (ND1, ND2, ND3, ND4, ND4L, ND5, ND6), ${ }^{36}$ and part of the glycolytic pathway (GAPDH). ${ }^{37}$

On the other hand, the underexpressed genes detected in the CTE and BD cases compared with normal controls included MIR3960, MIR149, UTF1, HSPA1A, IL10, and MYBL2. There is no distinct significant interaction between the group of genes compared with what can be seen in the normal population.

MIR3960 and MIR149 (microRNA 3960 and microRNA 149) have been known to be incorporated into RNAinduced silencing complex (RISC) and functioned as a translational inhibitor or destabilizer of the target mRNA. ${ }^{38,39}$

UTF1, or undifferentiated embryonic cell transcription factor, although mostly reported in pluripotent cells, was described as encoding a transcriptional repressor protein. $^{40}$

HSPA1A, or heat shock protein family A (Hsp70) member $1 \mathrm{~A}$, has been noted to prevent existing proteins from aggregation by stabilization and assist in protein folding in the cytosol and organelles. ${ }^{41}$

IL10, or interleukin 10, was characterized as encoding a cytokine that down-regulates Th1 cytokine and MHC class II antigen expression, while enhancing B cell survival, proliferation, and antibody production. It has been described to block NF-kappa B activity and has a co-stimulatory function on macrophages. ${ }^{42}$

MYBL2, or MYB proto-oncogene like 2, has been shown to encode for a protein with an activator function for $\mathrm{G} 2 / \mathrm{M}$ phase proteins such as Cyclin B 1, CDK 1, and Cyclin A2. ${ }^{43}$

All of the overexpressed and underexpressed genes observed in the CTE and BD cases had a significantly increased and decreased expression, respectively, in comparison with the normal subjects $(p<0.01)$. In contrast, only the overexpressed genes in PD have shown a significant difference with normal controls $(p<0.01)$, and the genes that showed an increase in expression in the normal controls, as compared with the CTE and BD cases with a $\log 2$ fold change of 0 , were also increased in the cases with PD.

The identification of parallel gene overexpression among the CTE, BD, and PD groups with respect to structural integrity, cellular metabolism, homeostasis, and apoptosis may indicate a common pathway that have been initiated as part of the response to maintain tissue function or as a consequence of the underlying pathobiologic mechanism that caused the primary lesion.

The presence of FAM107A among all the groups may imply the presence of related pathways among CTE, BD, and $\mathrm{PD}$ that have an impact to the regulation of synaptic function that are responsible for learning and long-term memory. This may also have a role on the other cognitive function that have a connection to the hippocampus.

On the other hand, the increased expression of the ferritin heavy chain 1 gene among the three groups may indicate the attempt of the brain to optimize neuroprotection and prevent oxidative damage since the presence of free iron may contribute to the increase in reactive oxygen species thru redox reactions and lead to the increased burden of cellular oxidative stress that may cause cell death. ${ }^{44}$

The presence of genes that are associated with mitochondrial function with respect to aerobic metabolism may indicate an increase in the general metabolic activity of the brain as a response to cellular injury and an attempt to facilitate repair. This is likewise seen in the context of an increased COX1/ PTGS1 and COX2 expression, which may be interpreted as a response to an inflammatory process and the need to increase the metabolic output to generate energy for the maintenance or restoration of homeostasis.

The similarities in structural integrity dysfunction that may be interpreted as a response to inflammation can be illustrated by the increase in MBP expression and such increase may indicate a response to a pathologic process that threatens the relative impermeability of the blood-brain barrier. The remaining genes such as CLU, 
HSP90AA1, MTURN, NDRG2, GLUL, and RNR1, may all have been increased to enable the production of proteins that ensure the maintenance of the tissue structure despite the injury. This may be further supported by the increase in EEF1A1, which plays a role in amino acid formation.

The presence of an apoptosis initiator in the form of GAPDH may indicate a selective facilitation of structures that require the cessation of processes due to an injured state. As such, the apoptosis may prevent further worsening of tissue inflammation by disrupting improper cellular functions that may lead to necrosis.

In comparison, the underexpressed genes detected in the CTE and BD cases compared to the normal controls and the PD cases may indicate the lack of genes that have a role in repressing the mRNA for protein coding such as MIR3960, MIR 149, AND UTF1. Interestingly, there is a decreased expression of an aggregation inhibitor gene, HSPA1A, which may have a role in preventing abnormal protein folding. The absence of this gene may be related to accumulation of abnormal protein structures that cause cellular destabilization and subsequent inflammation.

In terms of IL10 expression, mild CTE cases have been documented to have an increase in IL-10 cytokine. $^{45}$ However, murine studies have shown that the IL10 - / have higher superoxide production, are more susceptible to excitotoxicity, and neurotoxicity due to metabolic and hypoxic processes. ${ }^{46,47}$ Although there are contradictory statements regarding the role of IL-10 in $\mathrm{BD}$, the decreased expression of the IL10 gene may explain the imbalance in the pro-inflammatory and the anti-inflammatory cytokines that are associated with the adverse effects of neuroinflammation. Furthermore, a study indicating the presence of IL-10 cytokine only in the early stage of BD with subsequent loss of IL-10 during the later stages of $\mathrm{BD}$ after 10 or more years with the disease may explain the unmitigated neuroinflammation that is associated with BD brain lesions. ${ }^{48}$

On the other hand, MYBL2, or MYB proto-oncogene like 2, has been described in human gliomas as being associated with poorer prognosis. ${ }^{49}$ The underexpression of MYBL2 compared to controls may indicate the absence of uncontrolled astrocytic proliferation driver in the setting of tissue injury.

\section{CONCLUSION}

The overexpression of genes responsible for homeostasis, regulation of inflammation, balance of apoptosis and antiapoptosis, and maintenance of structural integrity among the CTE, BD, and PD groups indicate that there is an interrelated mechanism that serves converging pathways as part of the response to lesional-forming structures in the brain. Likewise, the underexpression of certain genes may indicate the lack of significant modifying proteins that may promote the silencing of genes that may contribute to the CNS pathology or increase modulating substances that can serve to mitigate the severity of the lesions. The role of studies such as this serves the primary purpose of paving the way for future large-scale studies that would correlate the clinical behavior of complex brain disorders with the histopathologic features and the gene expression profile. Although the impact of the research may be limited by the number of post-mortem brain samples, the analysis may provide an initial point of evaluation for further studies in comparative analysis. The goal of studies such as this is to have a better understanding on the common pathways that explain the interrelatedness of brain disorders and the putative mechanism for being co-morbid and pre-morbid conditions of each other. Furthermore, the study posits the challenge that attenuating the pre-morbid mechanisms that trigger the cascade of neuroinflammatory response may provide a means to delay the neural lesions that are accumulated in the late stages of the disorders.

\section{ACKNOWLEDGEMENTS}

The authors would like to thank the faculty and staff of the Department of Pathology and Pathology Laboratory of the UERM Memorial Medical Center.

\section{STATEMENT OF AUTHORSHIP}

All authors certified fulfillment of ICMJE authorship criteria.

\section{AUTHOR DISCLOSURE}

The authors declared no conflict of interest.

\section{FUNDING SOURCE}

None.

\section{REFERENCES}

1. Seo JS, Lee S, Shih JY, Hwang YJ, et al. Transcriptome analyses of chronic traumatic encephalopathy show alterations in protein phosphatase expression associated with tauopathy. Exp Mol Med. 2017;49(5):e333. PMID: 28524178. PMCID: PMC5454448. https://doi. org/10.1038/emm.2017.56.

2. Niu H, Shen L, Li T, Ren C, et al. Alpha-synuclein overexpression in the olfactory bulb initiates prodromal symptoms and pathology of Parkinson's disease. Transl Neurodegeneration. 2018;7:25. PMID: 30356861. PMCID: PMC6192070. https://doi. org/10.1186/s40035-018-0128-6.

3. Drange OK, Vaaler AE, Morken G, Andreassen OA, et al. Clinical characteristics of patients with bipolar disorder and premorbid traumatic brain injury: a cross-sectional study. Int J Bipolar Disord. 2018;6:19. PMID: 30198055. PMCID: PMC6162005. https://doi. org/10.1186/s40345-018-0128-6.

4. Faustino PR, Duarte GS, Chendo I, et al. Risk of Developing Parkinson Disease in Bipolar Disorder: A Systematic Review and Meta-analysis. JAMA Neurol. 2020;77(2). PMID: 31609378. PMCID: PMC6802493 (available on 2020-10-14). https://doi.org/10.1001/ jamaneurol.2019.3446.

5. Zhu S, Abounit S, Korth C, Zurzolo C. Transfer of disrupted-in-schizophrenia 1 aggregates between neuronal-like cells occurs in tunnelling nanotubes and is promoted by dopamine. Open Biol. 2017;7:160328. https://doi.org/10.1098/rsob.160328. 
6. Homo sapiens (human) Transcriptome analyses of chronic traumatic encephalopathy show alterations in protein phosphatase expression associated with tauopathy. Accession: PRJEB 13579. https://www.ncbi. nlm.nih.gov/bioproject/PRJEB 13579.

7. Homo sapiens (human) mRNA-Seq expression and MS3 proteomics profiling of human post-mortem BA9 brain tissue for Parkinson Disease and neurologically normal individuals. Accession:PRJNA283498. https:// www.ncbi.nlm.nih.gov/bioproject/PRJNA283498.

8. Homo sapiens (human) RNA-sequencing of the brain transcriptome implicates dysregulation of neuroplasticity, circadian rhythms, and GTPase binding in bipolar disorder. Accession: PRJNA231202. https:// www.ncbi.nlm.nih.gov/bioproject/PRJNA231202.

9. Galaxy. The Galaxy Project. 2020. https://usegalaxy. org/.

10. European Nucleotide Archive. European Molecular Biology Laboratory-European Bioinformatics Institute. 2020. https://www.ebi.ac.uk/ena.

11. GRCh38. Genome Reference Consortium Human Build 38. Genome Reference Consortium. https:// www.ncbi.nlm.nih.gov/assembly/GCF 000001405.39.

12. Kim D, Langmead B, Salzberg S. HISAT: a fast spliced aligner with low memory requirements. Nature Methods. 2015;12(4):357-60. https://doi.org/10.1038/ nmeth.3317.

13. Liao Y, Smyth GK, Shi W. featureCounts: an efficient general purpose program for assigning sequence reads to genomic features. Bioinformatics. 2013;30(7): 923-30. https://doi.org/10.1093/bioinformatics/btt656.

14. Gruening BA. Galaxy wrapper. 2014. https://github. com/bgruening/galaxytools.

15. RStudio. PBC. 2020. https://rstudio.com.

16. The $\mathrm{R}$ Foundation. The $\mathrm{R}$ Project for Statistical Computing. 2020. https://www.r-project.org/.

17. Szklarczyk D, Gable AL, Lyon D, et al. STRING v11: protein-protein association networks with increased coverage, supporting functional discovery in genomewide experimental datasets. Nucleic Acids Res. 2019 Jan; 47:D607-13. PMID: 30476243. PMCID: PMC6323986. https://doi.org/10.1093/nar/gky1131.

18. Nakajima H, Koizumi K. Family with sequence similarity 107: a family of stress responsive small proteins with diverse functions in cancer and the nervous system (Review). Biomed Rep. 2014;2(3):3215. PMID: 24748967. PMCID: PMC3990222. https:// doi.org/10.3892/br.2014.243.

19. Masana M, Westerholz S, Kretzschmar A, et al. Expression and glucocorticoid-dependent regulation of the stress-inducible protein DRR1 in the mouse adult brain. Brain Struct Funct. 2018;223(9):4039-52. PMID: 30121783. PMCID: PMC6267262. https://doi. org/10.1007/s00429-018-1737-7.

20. Jellinger K, Paulus W, Grundke-Iqbal I, et al. Brain iron and ferritin in Parkinson's and Alzheimer's diseases. J Neural Transm Gen Sect. 1990;2(4):327-40. PMID: 2078310. https://doi.org/10.1007/BF02252926.

21. Omar NN, Tash RF, Shoukry Y, El Saeed KO. Breaking the ritual metabolic cycle in order to save acetyl CoA: a potential role for mitochondrial humanin in $\mathrm{T} 2$ bladder cancer aggressiveness. J Egypt Natl Canc Inst. 2017;29(2):69-76. PMID: 28462847. https://doi. org/10.1016/j.jnci.2017.04.001.
22. Tarze A, Deniaud A, Le Bras M, Maillier E, et al. GAPDH, a novel regulator of the pro-apoptotic mitochondrial membrane permeabilization. 2007;26(18):2606-20. PMID: 17072346. https://doi. org/10.1038/sj.onc. 1210074 .

23. Zinkie S, Gentil BJ, Minotti S, Durham HD. Expression of the protein chaperone, clusterin, in spinal cord cells constitutively and following cellular stress, and upregulation by treatment with Hsp90 inhibitor. Cell Stress Chaperones. 2013;18(6):745-58. PMID: 23595219. PMCID: PMC3789872. https://doi. org/10.1007/s12192-013-0427-x

24. Zuehlke AD, Beebe $\mathrm{K}$, Neckers $\mathrm{L}$, Prince $\mathrm{T}$. Regulation and function of the human HSP90AA1 gene. Gene. 2015;570(1):8-16. PMID: 26071189. PMCID: PMC4519370. https://doi.org/10.1016/j. gene.2015.06.018.

25. Martinez-De Luna RI, Ku RY, Lyou Y, Zuber ME. Maturin is a novel protein required for differentiation during primary neurogenesis. Dev Biol. 2013;384(1): 26-40. PMID: 24095902. PMCID: PMC3843043. https://doi.org/10.1016/j.ydbio.2013.09.028.

26. Schonkeren SL, Massen M, van der Horst R, Koch A, Vaes N, Melotte V. Nervous NDRGs: the N-myc downstream-regulated gene family in the central and peripheral nervous system. Neurogenetics. 2019;20(4):173-86. PMID: 31485792. PMCID: PMC6754360. https://doi.org/10.1007/s10048-01900587-0.

27. Zhang R, Zhu JC, Hu H, Lin QY, Shao W, Ji TH. MicroRNA-140-5p suppresses invasion and proliferation of glioma cells by targeting glutamateammonia ligase (GLUL). Neoplasma. 2020;67(2): 371-8. PMID: 31986891. https://doi.org/10.4149/ neo_2020_190514N432.

28. Wolf NI, van Spaendonk RML, Hobson GM, et al (editors). In: GeneReviews ${ }^{\circledR}$ [Internet]. Seattle (WA): University of Washington, Seattle; 1993-2020. PLP1 Disorders. 1999 [updated 2019 Dec 19]. PMID: 20301361. NBK 1182.

29. Barbarino JM, McGregor TL, Altman RB, Klein TE. PharmGKB summary: very important pharmacogene information for MT-RNR1. Pharmacogenet Genomics. 2016;26(12):558-67. PMID: 27654872. PMCID: PMC5083147. https://doi.org/10.1097/FPC. 0000000000000247.

30. Namer IJ, Steibel J, Poulet P, Armspach JP, et al. Blood-brain barrier breakdown in MBP-specific $\mathrm{T}$ cell induced experimental allergic encephalomyelitis. A quantitative in vivo MRI study. Brain. 1993;116(Pt 1):147-59. PMID: 7680933. https://doi.org/10.1093/ brain/116.1.147.

31. Becker M, Kuhse J, Kirsch J. Effects of two elongation factor $1 \mathrm{~A}$ isoforms on the formation of gephyrin clusters at inhibitory synapses in hippocampal neurons. Histochem Cell Biol. 2013;140(6):603-9. PMID: 23839781. https://doi.org/10.1007/s00418-0131122-9.

32. Choi SH, Aid S, Bosetti F. The distinct roles of cyclooxygenase-1 and -2 in neuroinflammation: implications for translational research. Trends Pharmacol Sci. 2009;30(4):174-81. PMID: 19269697. PMCID: PMC3379810. NIHMSID: NIHMS385900. https://doi.org/10.1016/j.tips.2009.01.002. 
33. Jiang $T$, Cadenas E. Astrocytic metabolic and inflammatory changes as a function of age. Aging Cell. 2014;13(6):1059-67. PMID: 25233945. PMCID: PMC4244278. https://doi.org/10.1111/acel.12268.

34. Meunier B, Fisher N, Ransac S, Mazat JP, Brasseur G. Respiratory complex III dysfunction in humans and the use of yeast as a model organism to study mitochondrial myopathy and associated diseases. Biochim Biophys Acta. 2013;1827(11-12):1346-61. PMID: 23220121. https://doi.org/10.1016/j.bbabio. 2012.11.015.

35. Dautant A, Meier T, Hahn A, et al. ATP synthase diseases of mitochondrial genetic origin. Front Physiol. 2018;9:329. PMID: 29670542. PMCID: PMC5893901. https://doi.org/10.3389/fphys.2018.00329.

36. Kato T. The other, forgotten genome: mitochondrial DNA and mental disorders. Mol Psychiatry. 2001;6(6):625-33. PMID: 11673790. https://doi. org/10.1038/sj.mp.4000926.

37. Tristan C, Shahani N, Sedlak TW, Sawa A. The diverse functions of GAPDH: views from different subcellular compartments. Cell Signal. 2011;23(2):317-23. PMID: 20727968. PMCID: PMC3084531. https://doi. org 10.1016/j.cellsig.2010.08.003.

38. Ivashchenko A, Berillo O, Pyrkova A, Niyazova R, Atambayeva S. MiR-3960 binding sites with mRNA of human genes. Bioinformation. 2014;10(7):423-7. PMID: 25187682. PMCID: PMC4135290. https://doi. org/10.6026/97320630010423.

39. Shen X, Li J, Liao W, et al. microRNA-149 targets caspase-2 in glioma progression. Oncotarget. 2016;7(18):26388-99. PMID: 27049919. PMCID: PMC5041987. https://doi.org/10.18632/ oncotarget.8506.

40. Jia J, Zheng X, Hu G, et al. Regulation of pluripotency and self- renewal of ESCs through epigeneticthreshold modulation and mRNA pruning. Cell. 2012;151(3):576-89. PMID: 23101626. PMCID: PMC3575637. https://doi.org/10.1016/j.cell.2012.09. 023.

41. Deane CAS, Brown IR. Differential targeting of Hsp70 heat shock proteins HSPA6 and HSPA1A with components of a protein disaggregation/refolding machine in differentiated human neuronal cells following thermal stress. Front Neurosci. 2017;11:227. PMID: 28484369. PMCID: PMC5401876. https:// doi.org/10.3389/fnins.2017.00227.

42. Garcia JM, Stillings SA, Leclerc JL, et al. Role of Interleukin-10 in acute brain injuries. Front Neurol. 2017;8:244. PMID: 28659854. PMCID: PMC5466968. https://doi.org/10.3389/fneur.2017.00244.

43. Musa J, Aynaud MM, Mirabeau O, Delattre O, Grünewald TG. MYBL2 (B-Myb): a central regulator of cell proliferation, cell survival and differentiation involved in tumorigenesis. Cell Death Dis. 2017;8(6):e2895. PMID: 28640249. PMCID: PMC5520903. https://doi.org/10.1038/cddis.2017.244.

44. Daglas M, Adlard PA. The Involvement of iron in traumatic brain injury and neurodegenerative disease. Front Neurosci. 2018;12:981. PMID: 30618597. PMCID: PMC6306469. https://doi.org/10.3389/fnins. 2018.00981.

45. Lobo-Silva D, Carriche GM, Castro AG, Roque S, Saraiva M. Balancing the immune response in the brain: IL-10 and its regulation. J Neuroinflammation. 2016;297. https://doi.org/10.1186/s12974-016-0763-8.

46. Kinzenbaw DA, Chu Y, Peña Silva RA, Didion SP, Faraci FM. Interleukin-10 protects against aginginduced endothelial dysfunction. Physiol Rep. 2013; 1(6):e00149. PMID: 24400151. PMCID: PMC3871464. https://doi.org/10.1002/phy2.149.

47. Grilli M, Barbieri I, Basudev H, et al. Interleukin-10 modulates neuronal threshold of vulnerability to ischaemic damage. Eur J Neurosci. 2000;12(7):226572. PMID: 10947805. https://doi.org/10.1046/j.14609568.2000.00090.x

48. Barbosa IG, Bauer ME, Machado-Vieira R, Teixeira AL. Cytokines in bipolar disorder: paving the way for neuroprogression. Neural Plast. 2014;2014:360481. PMID: 25313338. PMCID: PMC4172873. https://doi. org/10.1155/2014/360481.

49. Zhang K, Fu G, Pan G, et al. Demethylzeylasteral inhibits glioma growth by regulating the miR-30e5p/MYBL2 axis. Cell Death Dis. 2018;9(10):1035. PMID: 30305611. PMCID: PMC6180101. https://doi. org/10.1038/s41419-018-1086-8.

Disclaimer: This journal is OPEN ACCESS, providing immediate access to its content on the principle that making research freely available to the public supports a greater global exchange of knowledge. As a requirement for submission to the PJP, all authors have accomplished an AUTHOR FORM, which declares that the ICMJE criteria for authorship have been met by each author listed, that the article represents original material, has not been published, accepted for publication in other journals, or concurrently submitted to other journals, and that all funding and conflicts of interest have been declared. Consent forms have been secured for the publication of information about patients or cases; otherwise, authors have declared that all means have been exhausted for securing consent. 